\title{
Translocation breakpoint at 7q31 associated with tics: further evidence for IMMP2L as a candidate gene for Tourette syndrome
}

\author{
Chirag Patel ${ }^{\star, 1}$, Lisa Cooper-Charles², Dominic J McMullan², Judith M Walker², Val Davison² and \\ Jenny Morton ${ }^{1}$
}

Gilles de la Tourette syndrome is a complex neuropsychiatric disorder with a strong genetic basis. We identified a male patient with Tourette syndrome-like tics and an apparently balanced de novo translocation $[46, \mathrm{XY}, \mathrm{t}(2 ; 7)(\mathrm{p} 24.2 ; \mathrm{q} 31)]$. Further analysis using array comparative genomic hybridisation (CGH) revealed a cryptic deletion at 7q31.1-7q31.2. Breakpoints disrupting this region have been reported in one isolated and one familial case of Tourette syndrome. In our case, IMMP2L, a gene coding for a human homologue of the yeast inner mitochondrial membrane peptidase subunit 2, was disrupted by the breakpoint on $7 q 31.1$, with deletion of exons 1-3 of the gene. The IMMP2L gene has previously been proposed as a candidate gene for Tourette syndrome, and our case provides further evidence of its possible role in the pathogenesis. The deleted region (7q31.1-7q31.2) of 7.2 $\mathrm{Mb}$ of genomic DNA also encompasses numerous genes, including FOXP2, associated with verbal dyspraxia, and the CFTR gene.

European Journal of Human Genetics (2011) 19, 634-639; doi:10.1038/ejhg.2010.238; published online 9 March 2011

Keywords: Tourette syndrome; IMMP2L; 7q31.1 region; array CGH; FOXP2; breakpoint mapping

\section{INTRODUCTION}

Gilles de la Tourette syndrome (GTS (MIM 137580)) is a neuropsychiatric disorder manifested by multiple, involuntary motor and vocal movements (tics) that fluctuate in severity. The motor tics are usually the initial symptom of the condition, with onset occurring usually between the ages of 2 and 14 years. Echolalia, grunting and coprolalia can develop as the disease progresses. GTS was previously considered to be a rare disorder, but population-based surveys estimate the prevalence at around 1/1000 males and 1/10 000 females. ${ }^{1}$ GTS has been reported with other behavioural disorders such as obsessive-compulsive disorder (OCD), attention-deficit hyperactivity disorder (ADHD) and conduct disorder-oppositional defiant disorder. ${ }^{2}$

It is thought that GTS is inherited in an autosomal dominant manner with variable expression and reduced penetrance, as around $10 \%$ of patients have a family history of the same condition. ${ }^{3}$ Several genome-wide linkage studies have failed to identify any candidate genes, but the largest genetic linkage study conducted so far in over 2000 individuals showed strong evidence of linkage at 2p23.2, 3p, 3q, 5p, 6p and 14q. ${ }^{4}$ Boghosian-Sell et al described a family affected by Tourette syndrome segregating with a balanced chromosomal translocation $[t(7 ; 18)]$. The Tourette syndrome in this family showed variable expression and reduced penetrance. ${ }^{5}$ No genes in the region of the breakpoint on chromosome 18 have been implicated in the aetiology of Tourette syndrome. The breakpoint on chromosome 7 was further mapped to $7 \mathrm{q} 22-\mathrm{q} 31$, between two genetic markers, D7S515 and D7S522. Kroisel et al ${ }^{6}$ reported on a 13-year-old boy with Tourette syndrome, minor physical anomalies and a de novo partial duplication of chromosome 7q $[\operatorname{dup}(7)(\mathrm{q} 22.1-\mathrm{q} 31.1)] .^{6}$ Molecular analysis of this case by Petek et a ${ }^{7}$ revealed that the duplication was inverted. The distal breakpoint occurred between the same two genetic markers that defined the region disrupted in the case by Boghosian-Sell et $a l,{ }^{5}$ raising the possibility of a candidate gene for GTS at $7 q 31$. Further studies identified that a novel gene, IMMP2L (MIM 605977), was interrupted by both the breakpoint in the duplicated fragment and the insertion site in $7 \mathrm{q} 31 .^{7}$

We describe the detailed molecular genetic analysis of an 18-yearold boy with a de novo translocation $\mathrm{t}(2 ; 7)(\mathrm{p} 24.2 ; \mathrm{q} 31)$ who developed motor tics at the age of 13 years and has significant speech and language impairment. The breakpoints are further characterised and the IMMP2L gene is found to be disrupted and partially deleted by the translocation, along with a cryptic 7.2-Mb deletion involving a number of genes.

\section{SUBJECTS AND METHODS \\ Case report}

The 18-year-old male propositus is the third child of healthy non-consanguineous White British parents. He was born at term with a birth weight of $2980 \mathrm{~g}$ (9-25th centiles). He sat unsupported at age 9 months and walked at age 17 months, later than his normal siblings. At age 3 years 6 months he had poor speech development and could utter only two-word sentences. His oromotor control was poor, resulting in constant severe dribbling. He attended a school for children with learning difficulties. At age 13 years he developed a movement disorder consisting of head and eye turning to the right, facial grimacing and puffing of his cheeks. He was partially able to control this, but it appears to be worsening with age. He was seen by a paediatric neurologist, who made a clinical diagnosis of tics rather than dystonia. He continues to have moderate

\footnotetext{
${ }^{1}$ Department of Clinical Genetics, Birmingham Women's Hospital NHS Foundation Trust, Birmingham, UK; ${ }^{2}$ West Midlands Regional Genetics Laboratory, Birmingham Women's Hospital NHS Foundation Trust, Birmingham, UK

*Correspondence: Dr C Patel, Department of Clinical Genetics, Birmingham Women's Hospital, Metchley Park Road, Edgbaston, Birmingham B15 2 TG, UK. Tel: +44 121 627 2630 ; Fax: +44 121627 2618; E-mail: Chirag.Patel@bwhct.nhs.uk
}

Received 10 August 2010; revised 1 November 2010; accepted 1 December 2010; published online 9 March 2011 
learning difficulties and severe speech difficulties, with verbal dyspraxia, and his speech is barely comprehensible to strangers. There is no family history of mental retardation, speech delay or movement disorders.

On examination at age 18 years his weight is between the 25 and 50th centiles and height between the 50 and 75th centiles. He has no facial dysmorphism, but has an intermittent divergent squint and hypoplastic 5th toenails. Neurological findings include the tics, normal tone and power in all four limbs, but brisk deep tendon reflexes, and a broad-based unsteady gait. An electroencephalogram showed a non-specific slight increase in background activity, but no focal or generalised seizure activity. A CT scan of the brain showed an enlarged cisterna magna, but was otherwise normal.

\section{Cytogenetic analysis}

Routine cytogenetic analyses were performed on metaphase chromosomes prepared from peripheral blood leukocytes using standard methods.

\section{Array studies}

Genomic DNA was extracted from peripheral blood using the AutoPure extraction system (Qiagen, Crawley, UK) following the manufacturer's instructions. DNA concentration and purity $\left(A_{260-280} \sim 1.8-2.0\right)$ were determined using a NanoDrop spectrophotometer (Labtech International, Ringmer, UK). Samples were labelled with Cy3 and Cy5 using a NimbleGen Dual Colour DNA Labelling kit (Roche NimbleGen, Madison, WI, USA) according to the manufacturer's instructions. In all, $500 \mathrm{ng}$ per patient genomic DNAs were hybridised to NimbleGen $12 \times 135 \mathrm{~K}$ arrays (Roche NimbleGen) and these arrays were washed post hybridisation according to the manufacturer's instructions. Arrays were scanned using a GenePix 4000B scanner and analysed in a Nexus Copy Number v3.0 (BioDiscovery, Inc., El Segundo, CA, USA), using the Rank Segmentation algorithm at default settings. Oligonucleotide probes were mapped to NCBI36.

\section{Fluorescent in situ hybridisation}

DNAs for bacterial artificial chromosome (BAC) fluorescent in situ hybridisation (FISH) probes were obtained from Roswell Park Cancer Institute, USA, and labelled in-house with Cy3 (GE Healthcare, Chalfont St Giles, UK) using the Bioprime labelling kit (Invitrogen Ltd, Paisley, UK). Hybridisation and washing were carried out using standard protocols. Images were visualised using an Olympus BX50 microscope and captured and processed using the Isis software package (Metasystems, Altlussheim, Germany).

\section{Multiplex ligation-dependent probe amplification}

Multiplex ligation-dependent probe amplification (MLPA) analysis of the CFTR gene was carried out using the MRC-Holland P091 kit (MRC-Holland, Amsterdam, The Netherlands) according to the manufacturer's instructions and using a Tetrad PCR machine (BioRad, Hercules, CA, USA). Capillary electrophoresis was carried out on an ABI 3130 and analysed using the Soft Genetics Gene Marker v1.70 program (SoftGenetics, State College, PA, USA).

\section{RESULTS}

\section{Identification of an apparently balanced translocation}

Cytogenetic analysis identified a reciprocal translocation between the short arm of chromosome 2 at band p24.2 and the long arm of chromosome 7 at band q31 (Figure 1). This was determined to be a de novo finding following analysis of parental samples. The chromosome 7 breakpoint occurred in the same band as a previously described duplication that disrupted the IMMP2L gene, and so this gene was implicated as a candidate for a role in the pathogenesis of GTS.?

\section{Identification of deletion at chromosome 7 breakpoint using array} CGH

Array analysis showed a 7.25-Mb deletion from $7 \mathrm{q} 31.1$ to $7 \mathrm{q} 31.2$ at the breakpoint of the $\mathrm{t}(2 ; 7)$ translocation. The deleted region contained 338 oligonucleotide probes genomic co-ordinates 7:110702484117947839 (Figures 2a and b). The proximal breakpoint of the



Figure 1 Partial G-banded karyogram showing $t(2 ; 7)(p 24.2 ; q 31)$ translocation (breakpoints arrowed).

deletion occurs within introns $2-3$ of the IMMP2L gene, and exons 1-3 are deleted. Exons 4-6 remain intact. The deleted region also contains 21 HGNC mapped genes (Table 1), including the OMIM Morbid entries FOXP2, MET and CFTR. Parental samples were not analysed using array CGH as the translocation was determined to be a de novo finding following analysis of parental chromosomes.

\section{FISH confirmation of partial deletion of IMMP2L}

FISH analysis using RP11-452K21 (co-ordinates 7:110396144110575013 , overlapping the $3^{\prime}$-end of IMMP2L) showed a signal present on both the derivative and normal chromosomes 7, confirming disruption of $I M M P 2 L$ and deletion of the $5^{\prime}$-end of the gene only (Figure 2c). RP11-148C1 (co-ordinates 7:110 833766-111014525, overlapping the $5^{\prime}$-end of IMMP2L) showed loss of signal on the derivative chromosome 7 (no movement of the signal to the derivative chromosome 2 was observed). Clear signal for this BAC was observed on the normal chromosome 7 , confirming deletion of the $5^{\prime}$-end of one copy of IMMP2L (Figure 2d). The FISH results therefore confirm the proximal breakpoint assigned by microarray analysis to lie between 110.58 and $110.83 \mathrm{Mb}$, within introns $2-3$ of IMMP2L.

\section{Comparison of copy number imbalance overlapping IMMP2L using} Ensembl and DECIPHER resources

Comparison of the deleted region in this case with other copy number imbalances involving the IMMP2L gene was carried out using the Database of Chromosomal Imbalance and Phenotype in Humans using Ensembl Resources (DECIPHER) (patient ID in DECIPHER: 248350). This identified nine additional cases with imbalance in this region only (DECIPHER entries 800, 801, 804, 813, 919, 1296, 1299, 1303 and 2391). Only one of these had motor and vocal tics as a clinical symptom (DECIPHER entry 919), which is the case reported by Petek et $a l^{7}$ of a duplication of $7 \mathrm{q} 22.1-7 \mathrm{q} 31.1$ with the distal breakpoint between exons 3 and 4 of IMMP2L. ${ }^{7}$ Of note, there are several entries of smaller copy number variation $(\mathrm{CNV})$ within the Database of Genomic Variants (DGV) within the IMMP2L gene (Figure 2b). Most of these encompass introns 2-3, but several of them also appear to include exons 1-3.

\section{Confirmation of deletion of the CFTR gene by MLPA}

Analysis of the CFTR gene using MLPA showed a heterozygous deletion of all exons of the gene at $7 \mathrm{q} 31.2$ confirming the array and 




b


C



d



Figure 2 (a) Chromosome $7 \log 2$ ratio displayed in Nexus Copy Number v3.0 showing 7.2 Mb deletion (indicated with red bar). (b) Zoomed proximal 7q31.1 region showing breakpoint at $110702484 \mathrm{Mbp}$ (red dotted line) within IMMP2L. Exons 1-2 and exons 1-3 are deleted from the IMMP2L-201 and IMMP2L-202 transcripts, respectively. (c) Confirmatory FISH analysis showing intact copies of RP11-452K21 (110.39-110.58 Mb). (d) Confirmatory FISH analysis showing deletion of one copy of RP11-148C1 (110.83-111.01 Mb). Red signals represent target BAC clones (locations arrowed) and green signals are control probe (CEP7, Abbott Vysis, Abbott Molecular, Maidenhead, UK). 
Table 1 HGNC mapped genes in the deletion interval $110702484-117947839$ (NBCl 36)

\begin{tabular}{|c|c|c|c|}
\hline Gene & Start $(M b p)$ & End $(M b p)$ & Description and aliases \\
\hline IMMP2L & 110090346 & 110948739 & IMP2 inner mitochondrial membrane peptidase-like (Saccharomyces cerevisiae). Aliases: IMP2 \\
\hline DOCK 4 & 111153404 & 111431423 & Dedicator of cytokinesis 4. Aliases: FLJ34238, KIAA0716 \\
\hline IFRDI & 111877751 & 111903480 & Interferon-related developmental regulator 1 . Aliases: PC4, TIS7 \\
\hline C7orf53 & 111908282 & 111918171 & Chromosome 7 open reading frame 53. Aliases: FLJ39575 \\
\hline TMEM168 & 112193032 & 112217714 & Transmembrane protein 168. Aliases: DKFZp564C012, FLJ13576 \\
\hline GPR85 & 112507704 & 112515015 & G-protein-coupled receptor 85. Aliases: SREB2 \\
\hline$P P P 1 R 3 A$ & 113301622 & 113346300 & Protein phosphatase 1 , regulatory (inhibitor) subunit $3 \mathrm{~A}$. Aliases \\
\hline FOXP2 & 113842288 & 114121063 & Forkhead box P2. Aliases: CAGH44 \\
\hline MDFIC & 114349445 & 114446501 & MyoD family inhibitor domain containing. Aliases: HIC \\
\hline TFEC & 115362448 & 115458103 & Transcription factor EC. Aliases: TCFEC, TFECL \\
\hline TES & 115637817 & 115686071 & Testis-derived transcript (3 LIM domains). Aliases: DKFZP586B2022, TESS-2, TESTIN \\
\hline CAV2 & 115926532 & 115935830 & Caveolin 2. Aliases: CAV \\
\hline CAV1 & 115952075 & 115988466 & Caveolin 1 , caveolae protein, $22 \mathrm{kDa}$. Aliases \\
\hline$M E T$ & 116099682 & 116225676 & Met proto-oncogene (hepatocyte growth factor receptor). Aliases: HGFR, RCCP2 \\
\hline CAPZA2 & 116289799 & 116346548 & Capping protein (actin filament) muscle Z-line, alpha 2. Aliases: CAPZ, CAPPA2 \\
\hline$S T 7$ & 116380617 & 116657393 & Suppression of tumorigenicity 7. Aliases: TSG7, SEN4, ETS7q, HELG, RAY1, FAM4A \\
\hline WNT2 & 116704518 & 116750579 & Wingless-type MMTV integration site family member 2. Aliases: IRP \\
\hline ASZ1 & 116790512 & 116854779 & Ankyrin repeat, SAM and basic leucine zipper domain containing 1. Aliases: Orf3, GASZ, ALP1 \\
\hline CFTR & 116907253 & 117095955 & Cystic fibrosis transmembrane conductance regulator (ATP-binding cassette sub-family $\mathrm{C}$, member 7 ) \\
\hline CTTNBP2 & 117137944 & 117300797 & Cortactin-binding protein 2. Aliases: KIAA1758, Orf4 \\
\hline LSM8 & 117611322 & 117620114 & LSM8 homologue, U6 small nuclear RNA associated (S. cerevisiae). Aliases: YJR022W \\
\hline ANKRD7 & 117651953 & 117669977 & Ankyrin repeat domain 7. Aliases: TSA806 \\
\hline
\end{tabular}

FISH results. The most centromeric of the probes shown to be deleted was located within the $A S Z 1$ gene that sits $58 \mathrm{~kb}$ upstream of the CFTR gene and the most telomeric probe deleted is located within the CTTNBP2 gene that sits $58 \mathrm{~kb}$ downstream of the CFTR gene (data not shown).

\section{DISCUSSION}

Chromosome abnormalities that segregate with a disease phenotype can facilitate the identification of disease loci and genes. Patients with an abnormal clinical phenotype and an apparently balanced de novo chromosome rearrangement on routine karyotyping have been shown to have submicroscopic imbalances on further analysis. Using CGH arrays, De Gregori et $a l^{8}$ found that $40 \%$ of patients with an abnormal phenotype and an apparently balanced translocation were in fact unbalanced. ${ }^{8}$ The majority of patients with Tourette syndrome have a normal karyotype and, of the various chromosomal abnormalities reported in association with Tourette syndrome, only the translocation cases reported by Boghosian-Sell et $a l^{5}$ and Kroisel et al ${ }^{6}$ show cosegregation with features associated with Tourette syndrome. ${ }^{5,6}$ Together, they provided evidence that a gene located in the breakpoint region at $7 \mathrm{q} 31$ could be involved in the development of Tourette syndrome. Previously reported cases with $7 \mathrm{q}$ duplications have not shown any signs of Tourette syndrome, which again supports the notion of involvement of gene(s) at the breakpoints rather than genes within the duplicated region (with haploinsufficiency as the mechanism).

Petek et $a l^{7}$ identified a novel gene, IMMP2L, disrupted by the breakpoints in their case of an inverted duplication of $7 \mathrm{q} 31$, and proposed this gene to be a candidate for GTS. ${ }^{7}$ The IMMP2L (inner mitochondrial membrane peptidase-2-like) gene spans $860 \mathrm{~kb}$ of genomic DNA, consisting of six exons encoding a 175 -amino-acid protein. The gene shows evolutionary conservation. The human protein is $41 \%$ identical to the yeast protein and $90 \%$ identical to the mouse protein. It is thought to function as a catalytic subunit of the mitochondrial inner membrane peptidase (IMP) complex. The IMP complex generates mature, active proteins in the mitochondrial intermembrane space by proteolytically removing the mitochondrial targeting pre-sequence of nuclear-encoded proteins. ${ }^{9}$ Although little is known about the exact function of the protein, of note, other mitochondrial proteins (eg, COXII) have been shown to be involved in neurodegenerative and neuropsychiatric disorders. ${ }^{10-12}$

Family-based association studies by Díaz-Anzaldúa et $a l^{13}$ in a French-Canadian GTS sample showed significant association with the region previously described by Petek et $a l^{7}$ in their case with the inverted duplication of $7 \mathrm{q} 31$. They found a significant association close to the first duplication breakpoint with markers D7S523 and D7S522 (situated 1.2 and $5 \mathrm{Mb}$ distal to this breakpoint), and marker D7S1516 (situated within the IMMP2L gene). ${ }^{13}$ A further study in 2007 by Petek et al screening 39 unrelated patients with GTS, for mutations in IMMP2L, detected no coding-region variants, and so failed to provide further evidence that $I M M P 2 L$ mutations are a common cause of GTS. However, the gene may be involved at a more subtle level or in just a small proportion of cases. They concluded that to exclude this gene from a pathogenic role in GTS, studies screening for mutations in the intronic promoter, enhancer and other regulatory elements is required. ${ }^{14}$

A number of cases of deletions and duplications overlapping the $7 \mathrm{q} 31.1$ region (and including IMMP2L) have been reported in the DECIPHER database but only one case has documented vocal and motor tics (DECIPHER entry 919) as part of the phenotype. There could be a number of explanations for this. First, some of these cases are younger than 3 years old and may be too young to have yet developed features of the condition. Both our case and that reported by Kroisel et $a l^{6}$ developed Tourette syndrome after age 11 years. Second, the absence of GTS could also be explained by the variable penetrance of the condition. Lastly, the cases in DECIPHER do not involve disruption of $I M M P 2 L$, but all involve complete deletion leading to haploinsufficiency for IMMP2L. Our case (with deletion of exons 1-3 out of 6) and the translocation case studied by Petek et $a l^{7}$ provide evidence of disruption of IMMP2L. A truncated gene and the inability to transcribe a full-length mRNA, along with the 
production of a defective protein, may be responsible for the appearance of features of GTS, and our case provides further evidence for this. This may be an important pathogenetic mechanism rather than deletion or duplication of the entire gene. Reported cases of $\mathrm{CNV}$ within IMMP2L also exist in the DGV, although, given the variable penetrance associated with GTS and the lack of phenotypic data available for 'healthy control cohorts' generally assessed for studies of 'normal' variation recorded in the DGV, it is not possible to exclude the involvement of IMMP2L in GTS on the basis of these cases. These points also highlight the importance of follow-up of all cases with overlapping deletions and duplications encompassing IMMP2L gene, to allow not only early detection and management of features of GTS, but also further understanding of the genetic mechanisms by which the IMMP2L gene is implicated in the pathogenesis of GTS.

Our case has significant verbal dyspraxia and speech difficulties. He sometimes omits the end sound in words and has difficulty in producing the sound of some consonants. He has problems in producing words with three or more syllables, especially when used in sentences and conversation. Array CGH analysis in our case identified the FOXP2 gene (MIM 605317) to be completely deleted. The FOXP2 gene encodes a transcription factor thought to regulate gene expression in defined areas of the developing neural, lung, cardiovascular and intestinal tissue, and was the first gene to be implicated in a developmental communication disorder. Abnormalities of FOXP2 are associated with difficulties in the learning and production of sequences of oral movement, which impair speech, known as developmental verbal dyspraxia. Affected individuals have variable levels of impairment in expressive and receptive language, sometimes extending to problems with production and comprehension of grammar. Hurst et al $l^{15}$ described a large multigenerational family with a severe speech and language disorder inherited in an autosomal dominant manner. ${ }^{15} \mathrm{~A}$ similar speech and language disorder was presented by Lai $e a^{16}$ in a patient with a de novo balanced reciprocal translocation $\mathrm{t}(5 ; 7)(\mathrm{q} 22 ; \mathrm{q} 31.2){ }^{16}$ They mapped the $7 \mathrm{q} 31.2$ breakpoint to a single clone, containing several exons of the FOXP2 gene, showing disruption of this gene in their case. They also identified a point mutation in the FOXP2 gene in the family reported by Hurst et $a l,{ }^{15}$ which co-segregated perfectly with the speech and language disorder in this family. ${ }^{17}$ Case reports of deletions within the $7 \mathrm{q} 31$ region including FOXP $2{ }^{18}$ and studies showing mutations within FOXP2 in isolated cases of verbal dyspraxia, ${ }^{19}$ have further supported the evidence of this gene being important for normal speech and language development. It is very likely that the speech impairment, verbal dyspraxia, and possibly oropharyngeal dyspraxia and dribbling seen in our case are due to haploinsufficiency of this gene.

The IMMP2L gene (along with numerous other neurodevelopmental genes) has also recently been implicated in structural variation studies carried out on cohorts of patients with autistic spectrum disorder, ${ }^{20}$ and ADHD. ${ }^{21}$ Our case does not display any of the classical features of autism, except speech impairment (which is likely to be accounted for by deletion of the FOXP2 gene) or ADHD. Autism or autistic spectrum disorder (ASD (MIM 209850)) is a severe neurodevelopmental disorder that presents at an early age and persists throughout life. Individuals display impairments in social interaction and communication coupled with repetitive and/or obsessive-compulsive behaviours. Data from family and twin studies suggest a strong genetic component, with the likelihood of numerous genes contributing to the pathogenesis in an additive manner. Genetic and cytogenetic studies have provided evidence to suggest one or more autism susceptibility genes within the $7 \mathrm{q} 22-\mathrm{q} 31$ region. ${ }^{22}$ Hutcheson et $a^{23}$ further defined this region to be a $3-\mathrm{cM}$ region on $7 \mathrm{q} 31-\mathrm{q} 33$ between markers D7S496 and D7S523, containing several interesting functional candidate genes, including some neuronally expressed genes: NRCAM (MIM 601581) and LRRN3. ${ }^{23}$ Neither of these genes is within our deleted region, but LRRN3 is located centromeric to the proximal deletion breakpoint and very near the last few exons of the IMMP2L gene.

Our case does have moderate mental retardation and this could be accounted for by haploinsufficiency of an as yet unknown mental retardation gene within the deleted region. Some of the genes within the deleted region may be good candidate genes for a role in the pathogenesis of mental retardation (eg, DOCK4, GPR85, MET, WNT2 and CTTNBP2). The DOCK4 gene (MIM 607679) has been shown to regulate dendritic growth in neuronal cell lines in rats. ${ }^{24} \mathrm{~A}$ recent casecontrol study in families linked to the autism susceptibility locus 1 (AUTS1) detected single-nucleotide polymorphisms (SNPs) and CNV within DOCK4 and IMMP2L, implicating them in autism susceptibility. ${ }^{20}$ A DOCK4 deletion segregating in a family with dyslexia, and undetectable in $>2500$ controls, suggested that DOCK4 may have a role in the aetiology of dyslexia. ${ }^{25}$ The GPR85 gene (MIM 605188) is predominantly expressed in the central nervous system, especially in structures exhibiting high levels of plasticity (eg, hippocampal dentate gyrus); it has high evolutionary conservation across three mammalian species, ${ }^{26}$ and has been implicated as a potential risk factor for psychiatric disorders. ${ }^{27}$ The MET gene (MIM 164860) encodes for a receptor tyrosine kinase and participates in neocortical and cerebellar growth and maturation, as well as immune and gastrointestinal functions. In 204 families with autism, Campbell et al found a significant association between autism and a G-C transversion in the promoter of the MET gene. Functional assays showed that the $\mathrm{C}$ allele resulted in a two-fold decrease in $M E T$ promoter activity and altered binding of specific transcription factor complexes. ${ }^{28}$ The WNT family of genes influences the development of the central nervous system, and a mouse knockout of the DVLI gene (a member of a gene family essential for the function of the WNT pathway) exhibited a behavioural phenotype characterised by diminished social interaction. Wassink et $a l^{29}$ screened the WNT2 gene (MIM 147870) in a large number of autistic probands and found two families containing a non-conservative coding sequence variant that segregated with autism in those families. ${ }^{29}$ Cheung et $\mathrm{ll}^{30}$ showed high levels of expression of CTTNBP2 (MIM 609772) in the brain and screened it for mutations in 90 unrelated individuals with autism, and identified two variants. ${ }^{30}$ It is possible though that speech and language impairment and autistic traits may be influenced by a single gene or multiple interacting genes within this region.

The CFTR gene (MIM 602421) is also located within the deleted region in our patient, therefore making him a carrier of cystic fibrosis (CF) (MIM 219700). This will have implications for future offspring and the necessity to offer carrier testing to any future partners. This case, in addition to providing supportive evidence for disruption of $I M M P 2 L$ gene function causing GTS, has highlighted some very important genetic counselling issues for the patient: (1) standard 'balanced' translocation (and the risk of larger chromosomal imbalance in any offspring), (2) offspring risk for GTS, (3) offspring risk for mental retardation, (4) offspring risk for verbal dyspraxia and (5) implications of being a CF carrier.

\section{CONCLUSION}

Our case adds to evidence from previous reports of the importance of genes within the $7 \mathrm{q} 31$ region, particularly the association of IMMP2L with GTS, and FOXP2 with verbal dyspraxia. There is evidence for considerable genetic heterogeneity in GTS, and the number of cases 
attributable to abnormalities of IMMP2L is yet to be determined. Further studies are required to determine the role of $I M M P 2 L$ in the aetiology of GTS. We also highlight the value of array CGH in obtaining a cytogenetic diagnosis in an apparently balanced translocation with an abnormal phenotype, and the identification of new candidate genes.

\section{ACKNOWLEDGEMENTS}

We are particularly grateful to the parents of the case who supported our publication of their son's case to benefit other families.

\section{CONFLICT OF INTEREST}

The authors declare no conflicts of interest.

\section{WEB RESOURCES}

Database of Chromosomal Imbalance and Phenotype in Humans using Ensembl Resources (DECIPHER), http://decipher.sanger.ac.uk Database of Genomic Variants (DGV), http://projects.tcag.ca/variation/ Ensemble, http://www.ensembl.org/index.html

Online Mendelian Inheritance in Man (OMIM), http://www.ncbi.nlm.nih.gov/ OMIM/.

1 Burd L, Kerbeshian J, Wikenheiser M, Fisher W: A prevalence study of Gilles de la Tourette syndrome in North Dakota school-age children. J Am Acad Child Psychiatry 1996; 25: 552-553.

2 Freeman RD, Fast DK, Burd L, Kerbeshian J, Robertson MM, Sandor P: An international perspective on Tourette syndrome: selected findings from 3,500 individuals in 22 countries. Dev Med Child Neurol 2000; 42: 436-447.

3 Pauls DL, Raymond CL, Stevenson JM, Leckman JF: A family study of Gilles de la Tourette syndrome. Am J Hum Genet 1991; 48: 154-163.

4 Tourette Syndrome Association International Consortium for Genetics: Genome scan for Tourette disorder in affected-sibling-pair and multigenerational families. Am J Hum Genet 2007; 80: 265-272.

5 Boghosian-Sell L, Comings DE, Overhauser J: Tourette syndrome in a pedigree with a 7;18 translocation: identification of a YAC spanning the translocation breakpoint at 18q22.3. Am J Hum Genet 1996; 59: 999-1005.

6 Kroisel PM, Petek E, Emberger W, Windpassinger C, Wladika W, Wagner K: Candidate region for Gilles de la Tourette syndrome at 7q31. Am J Med Genet 2001; 101: 259-261.

7 Petek E, Windpassinger C, Vincent JB et al: Disruption of a novel gene (IMMP2L) by a breakpoint in 7q31 associated with Tourette syndrome. Am J Hum Genet 2001; 68: 848-858.

8 De Gregori M, Ciccone R, Magini $\mathrm{P}$ et al: Cryptic deletions are a common finding in 'balanced' reciprocal and complex chromosome rearrangements: a study of 59 patients. J Med Genet 2007; 44: 750-762.

9 Burri L, Strahm Y, Hawkins CJ et al: Mature DIABLO/Smac is produced by the IMP protease complex on the mitochondrial inner membrane. Mol Biol Cell 2005; 16: 2926-2933.
10 Whatley SA, Curti D, Marchbanks RM: Mitochondrial involvement in schizophrenia and other functional psychoses. Neurochem Res 1996; 21: 995-1004.

11 Graeber MB, Müller U: Recent developments in the molecular genetics of mitochondrial disorders. J Neurol Sci 1998; 153: 251-263.

12 Leonard JV, Schapira AH: Mitochondrial respiratory chain disorders II: neurodegenerative disorders and nuclear gene defects. Lancet 2000; 355: 389-394.

13 Díaz-Anzaldúa A, Joober R, Rivière JB et al: Association between 7q31 markers and Tourette syndrome. Am J Med Genet 2004; 127A: 17-20.

14 Petek E, Schwarzbraun T, Noor A et al: Molecular and genomic studies of IMMP2L and mutation screening in autism and Tourette syndrome. Mol Genet Genomics 2007; 277 . 71-81.

15 Hurst JA, Baraitser M, Auger E, Graham F, Norell S: An extended family with a dominantly inherited speech disorder. Dev Med Child Neurol 1990; 32. 352-355

16 Lai CS, Fisher SE, Hurst JA et al: The SPCH1 region on human 7q31: genomic characterization of the critical interval and localization of translocations associated with speech and language disorder. Am J Hum Genet 2000; 67: 357-368.

17 Lai CS, Fisher SE, Hurst JA, Vargha-Khadem F, Monaco AP: A forkhead-domain gene is mutated in a severe speech and language disorder. Nature 2001; 413: 519-523.

18 Lennon PA, Cooper ML, Peiffer DA et al: Deletion of 7q31.1 supports involvement of FOXP2 in language impairment: clinical report and review. Am J Med Genet 2007; 143A: 791-798.

19 MacDermot KD, Bonora $\mathrm{E}$, Sykes $\mathrm{N}$ et al: Identification of FOXP2 truncation as a nove cause of developmental speech and language deficits. Am J Hum Genet 2005; 76: 1074-1080.

20 Maestrini E, Pagnamenta AT, Lamb JA et al: High-density SNP association study and copy number variation analysis of the AUTS1 and AUTS5 loci implicate the IMMP2LDOCK4 gene region in autism susceptibility. Mol Psychiatry 2010; 15: 954-968.

21 Elia J, Gai X, Xie HM et al: Rare structural variants found in attention-deficit hyperactivity disorder are preferentially associated with neurodevelopmental genes. Mol Psychiatry 2010; 15: 637-646.

22 International Molecular Genetic Study of Autism Consortium: A full genome screen for autism with evidence for linkage to a region on chromosome 7q. Hum Mol Genet 1998; 7: $571-578$

23 Hutcheson HB, Bradford Y, Folstein SE et al: Defining the autism minimum candidate gene region on chromosome 7. Am J Med Genet 2003; 117B: 90-96.

24 Ueda S, Fujimoto S, Hiramoto K, Negishi M, Katoh H: Dock4 regulates dendritic development in hippocampal neurons. J Neurosci Res 2008; 86: 3052-3061

25 Pagnamenta AT, Bacchelli $\mathrm{E}$, de Jonge MV et al: Characterization of a family with rare deletions in CNTNAP5 and DOCK4 suggests novel risk loci for autism and dyslexia. Biol Psychiatry 2010; 68: 320-328.

26 Matsumoto M, Beltaifa S, Weickert CS et al: A conserved mRNA expression profile of SREB2 (GPR85) in adult human, monkey, and rat forebrain. Brain Res Mol Brain Res 2005; 138: 58-69.

27 Matsumoto M, Straub RE, Marenco $S$ et al: The evolutionarily conserved G proteincoupled receptor SREB2/GPR85 influences brain size, behaviour, and vulnerability to schizophrenia. Proc Natl Acad Sci USA 2008; 105: 6133-6138.

28 Campbell DB, Sutcliffe JS, Ebert PJ et al: A genetic variant that disrupts MET transcription is associated with autism. Proc Natl Acad Sci USA 2006; 103 : 16834-16839.

29 Wassink TH, Piven J, Vieland VJ et al: Evidence supporting WNT2 as an autism susceptibility gene. Am J Med Genet 2001; 105: 406-413.

30 Cheung J, Petek E, Nakabayashi K, Tsui LC, Vincent JB, Scherer SW: Identification of the human cortactin-binding protein-2 gene from the autism candidate region at $7 \mathrm{q} 31$. Genomics 2001; 78: 7-11. 